\title{
Wave damping by a thin layer of viscous fluid
}

\author{
Alastair D. Jenkins \\ Nansen Environmental and Remote Sensing Center, Edvard Griegs vei 3a, N-5037 Solheimsviken, \\ Bergen, Norway \\ Stanley J. Jacobs \\ Department of Atmospheric, Oceanic and Space Sciences, 1538 Space Research Building, \\ The University of Michigan, Ann Arbor, Michigan 48109-2143
}

(Received 22 March 1996; accepted 7 January 1997)

\begin{abstract}
The rate of damping of surface gravity-capillary waves is investigated, in a system which consists of a thin layer of a Newtonian viscous fluid of thickness $d$ floating on a Newtonian fluid of infinite depth. The surface and interfacial tensions, elasticities and viscosities are taken into account. In particular, an approximate dispersion relation is derived for the case where $k d$ and $\left(\omega / \nu_{+}\right)^{1 / 2} d$ are both small, where $k$ is the wavenumber, $\omega$ is the angular frequency and $\nu_{+}$is the kinematic viscosity of the upper fluid. If $d \rightarrow 0$ while $\nu_{+} d$ remains finite, published dispersion relations for viscoelastic surface films of extremely small (e.g., monomolecular) thickness are reproduced, if we add the surface and interfacial tensions, elasticities and viscosities together, and then add an additional $4 \rho_{+} \nu_{+} d$ to the surface viscosity, where $\rho_{+}$is the density of the upper fluid. A simple approximation is derived for the damping rate and associated frequency shift when their magnitudes are both small. An example is given of what may happen with a slick of heavy fuel oil on water: a slick $10 \mu \mathrm{m}$ thick produces a damping rate only slightly different from that of a film of essentially zero thickness, but the effect of the finite thickness becomes very noticeable if it is increased to 0.1-1 mm. (C) 1997 American Institute of Physics. [S1070-6631(97)00905-7]
\end{abstract}

\section{INTRODUCTION}

The hydrodynamic theory of wave damping by viscoelastic films of negligible thickness (e.g., monomolecular films) on the surface of a Newtonian fluid is well established, ${ }^{1-9}$ the dominant effect being the increase in the rate of damping by a factor of order $\left(\nu k^{2} / \omega\right)^{-1 / 2}$ over the clean-surface value ${ }^{10-12}$ of $2 \nu k^{2}$, where $\nu$ is the kinematic viscosity, $k$ is the wavenumber ( $k=2 \pi /$ wavelength), and $\omega$ is the angular frequency. This effect is due to an increase in velocity shear in the viscous surface boundary layer of thickness $(2 \nu / \omega)^{1 / 2}$, as a result of the altered boundary condition for the tangential stress component.

Wave damping due to the presence of a viscous surface fluid layer of finite thickness is also of practical importance, for example, in the initial stages of an oil spill. ${ }^{13-16}$ An improved knowledge of the rates of wave damping for oil slicks of finite thickness may help to distinguish oil spills from other low-backscatter features in satellite and airborne radar images. ${ }^{17-22}$ Wave damping by a finite-thickness surface layer has been investigated mathematically by Weber, ${ }^{23}$ who applied his results to the damping of waves by a layer of grease ice.

\section{GOVERNING EQUATIONS AND BOUNDARY CONDITIONS}

We consider motions in two dimensions, $x$ (horizontal) and $z$ (vertical), of a system consisting of two fluid layers: an upper layer of thickness $d$ floating on a lower fluid body of infinite depth. The horizontal and vertical velocity components are $U$ and $W$, respectively, and we employ a reduced pressure variable $P=(\varpi / \rho)+g z-g\left(\rho_{+} / \rho\right) d$, where $\varpi$ is the actual pressure, $\rho$ is the density (in either fluid), $\rho_{+}$is the density of the upper fluid, and $g$ is the acceleration due to gravity. Subscripts indicate partial differentiation with respect to the coordinates and time $t$. We assume that the nonlinear terms in the Navier-Stokes equations for both the upper layer and the lower bulk fluid can be neglected in comparison with the acceleration terms $U_{t}$ and $W_{t}$, for both the upper layer and the lower bulk fluid: this is the case for surface waves in the limit that $a k \ll 1$, where $a$ is the wave amplitude. For a further discussion of nonlinear effects and the validity of the linearization procedure, we refer the reader to Appendix A. In particular, the linear approximation is shown to be valid for $a k \ll 1$ even where $a \gg d$.

In the notation employed below, the subscript $(\cdot)_{+}$refers to either the upper fluid layer or to the surface, where appropriate, and $(\cdot)_{-}$refers correspondingly to the lower fluid or the interface. The linearized continuity and momentum equations are

$$
\begin{aligned}
& U_{x}+W_{z}=0, \\
& U_{t}+P_{x}=\nu \nabla^{2} U, \\
& W_{t}+P_{z}=\nu \nabla^{2} W .
\end{aligned}
$$

We let the interface between the two fluids be at $z=H$ and the free surface be at $z=d+Z$, the corresponding undisturbed levels being $z=0$ and $z=d$, respectively. At the free surface, the kinematic boundary condition is

$$
W=Z_{t},
$$

the tangential stress condition is ${ }^{1-7}$

$$
\rho_{+} \nu_{+}\left(U_{z}+W_{x}\right)=\chi_{+} \xi_{x x}+\nu_{s+} \xi_{x x t},
$$

and the normal stress condition is

$$
P-g Z-2 \nu_{+} W_{z}+\left(\gamma_{+} / \rho_{+}\right) Z_{x x}=0,
$$


where $\gamma_{+}$is the surface tension, $\chi_{+}$is the surface elasticity, and $\nu_{s+}$ is the surface viscosity. The quantity $\xi$ is the horizontal fluid particle displacement, which satisfies

$$
U=\xi_{t} \text {. }
$$

At the interface, the kinematic boundary condition is

$$
W_{+}=W_{-}=H_{t},
$$

continuity of the velocity field gives

$$
U_{+}=U_{-},
$$

and the tangential and normal stress conditions are

$$
\begin{gathered}
\rho_{+} \nu_{+}\left(U_{+_{z}}+W_{+_{x}}\right)+\chi_{-} \xi_{x x}+\nu_{s-} \xi_{x x t} \\
=\rho_{-} \nu_{-}\left(U_{-_{z}}+W_{-_{x}}\right)
\end{gathered}
$$

and

$$
\begin{aligned}
& \rho_{+}\left(P_{+}-g H-2 \nu_{+} W_{+_{z}}\right) \\
& \quad=\rho_{-}\left(P_{-}-g H-2 \nu_{-} W_{-z}\right)+\gamma_{-} H_{x x},
\end{aligned}
$$

where $\gamma_{-}, \chi_{-}$and $\nu_{s_{-}}$are the interfacial tension, elasticity, and viscosity, respectively. We assume that the motions decay to zero as $z \rightarrow-\infty$.

Now assume that we have travelling waves in the $x$-direction, so that

$$
(U, W, P, Z, H)=(u(z), w(z), p(z), \zeta, \eta) e^{i k x+n t},
$$

where $n=-i \omega$. We now choose nondimensional variables and parameters

$$
\begin{aligned}
& \left(x^{*}, z^{*}, d^{*}, \zeta^{*}, \eta^{*}, \xi^{*}\right)=(x, z, d, \zeta, \eta, \xi) k, \\
& t^{*}=(g k)^{1 / 2} t, \\
& k^{*}=1, \quad\left(n^{*}, \omega^{*}\right)=(g k)^{-1 / 2}(n, \omega),
\end{aligned}
$$

$$
\begin{aligned}
& \left\{2 \cosh d+\left(l_{+}{ }^{2}+1\right) \cosh \left(l_{+} d\right)+\frac{\nu_{E+}\left(l_{+}{ }^{2}-1\right)}{\rho_{+} n}\left[\sinh d+l_{+} \sinh \left(l_{+} d\right)\right]\right\} c_{c+}+\left\{2 \cosh d-\left(l_{+}{ }^{2}+1\right) \cosh \left(l_{+} d\right)\right. \\
& \left.\quad+\frac{\nu_{E+}\left(l_{+}{ }^{2}-1\right)}{\rho_{+} n}\left[\sinh d-l_{+} \sinh \left(l_{+} d\right)\right]\right\} c_{c_{-}}+\left\{2 \sinh d+l_{+}{ }^{-1}\left(l_{+}{ }^{2}+1\right) \sinh \left(l_{+} d\right)\right. \\
& \left.\quad+\frac{\nu_{E+}\left(l_{+}{ }^{2}-1\right)}{\rho_{+} n}\left[\cosh d+l_{+} \cosh \left(l_{+} d\right)\right]\right\} c_{s_{+}}+\left\{2 \sinh d-l_{+}{ }^{-1}\left(l_{+}{ }^{2}+1\right) \sinh \left(l_{+} d\right)\right. \\
& \left.\quad+\frac{\nu_{E+}\left(l_{+}{ }^{2}-1\right)}{\rho_{+} n}\left[\cosh d-l_{+} \cosh \left(l_{+} d\right)\right]\right\} c_{s-}=0 \\
& \left\{\frac{\rho_{+} n^{2}}{\left(l_{+}{ }^{2}-1\right)^{2}}\left[\left(l_{+}{ }^{2}+1\right) \sinh d+2 l_{+} \sinh \left(l_{+} d\right)\right]+\left(\rho_{+}+\gamma_{+}\right)\left[\cosh d+\cosh \left(l_{+} d\right)\right]\right\} c_{c+} \\
& \quad+\left\{\frac{\rho_{+} n^{2}}{\left(l_{+}{ }^{2}-1\right)}\left[\left(l_{+}{ }^{2}+1\right) \sinh d-2 l_{+} \sinh \left(l_{+} d\right)\right]+\left(\rho_{+}+\gamma_{+}\right)\left[\cosh d_{-} \cosh \left(l_{+} d\right)\right]\right\} c_{c-} \\
& \quad+\left\{\frac{\rho_{+} n^{2}}{\left(l_{+}{ }^{2}-1\right)}\left[\left(l_{+}{ }^{2}+1\right) \cosh d+2 \cosh \left(l_{+} d\right)\right]+\left(\rho_{+}+\gamma_{+}\right)\left[\sinh d+l_{+}{ }^{-1} \sinh \left(l_{+} d\right)\right]\right\} c_{s+} \\
& \quad+\left\{\frac{\rho_{+} n^{2}}{\left(l_{+}{ }^{2}-1\right)}\left[\left(l_{+}{ }^{2}+1\right) \cosh d-2 \cosh \left(l_{+} d\right)\right]+\left(\rho_{+}+\gamma_{+}\right)\left[\sinh d-l_{+}{ }^{-1} \sinh \left(l_{+} d\right)\right]\right\} c_{s-}=0
\end{aligned}
$$




$$
\begin{aligned}
& c_{c+}-c_{1}-c_{3}=0, \\
& c_{s+}-c_{1}-l c_{3}=0, \\
& \frac{1}{2} \rho_{+} n \frac{l_{+}{ }^{2}+3}{l_{+}{ }^{2}-1} c_{c+}-\frac{1}{2} \rho_{+} n c_{c-}+\nu_{E-} c_{s+}-\frac{2 n}{l^{2}-1} c_{1} \\
& \quad-n \frac{l^{2}+1}{l^{2}-1} c_{3}=0, \\
& -\rho_{+} c_{c+}-\frac{1}{2} \rho_{+} n^{2} \frac{l_{+}{ }^{2}+3}{l_{+}{ }^{2}-1} c_{s+}-\frac{1}{2} \rho_{+} n^{2} c_{s-} \\
& \quad+\left(n^{2} \frac{l^{2}+1}{l^{2}-1}+1+\gamma_{-}\right) c_{1}+\left(\frac{2 \ln { }^{2}}{l^{2}-1}+1+\gamma_{-}\right) c_{3}=0
\end{aligned}
$$

where we have introduced the effective (complex) ${ }^{24}$ surface/ interface viscosity $\nu_{E \pm}=\chi_{ \pm} / n+\nu_{s \pm}$.

\section{DISPERSION RELATION AND WAVE DAMPING}

\section{A. General case}

The system of equations (17)-(22) has a non-trivial solution if the determinant of the coefficients is zero, from which condition we can derive the dispersion relation and hence, from the real part of $n$, the rate of wave damping. We can in fact eliminate $c_{c \pm}$ and $c_{s \pm}$ to obtain a system of two equations in the two unknowns $c_{1}$ and $c_{3}$, and equate the corresponding $2 \times 2$ determinant to zero.

From (19)-(22) we obtain $c_{c-}$ and $c_{s-}$ in terms of $c_{1}$ and $c_{3}$ :

$$
\begin{aligned}
c_{c-}= & {\left[\frac{l_{+}{ }^{2}+3}{l_{+}{ }^{2}-1}-\frac{4}{\rho_{+}\left(l^{2}-1\right)}+\frac{2 \nu_{E-}}{\rho_{+} n}\right] c_{1}+\left[\frac{l_{+}{ }^{2}+3}{l_{+}{ }^{2}-1}\right.} \\
& \left.-\frac{2}{\rho_{+}}\left(\frac{l^{2}+1}{l^{2}-1}\right)+\frac{2 \nu_{E-} l}{\rho_{+} n}\right] c_{3}, \\
c_{s-}= & {\left[-2 \frac{\rho_{+}+\gamma_{+}}{\rho_{+} n^{2}}-\frac{l_{+}{ }^{2}+3}{l_{+}{ }^{2}-1}+\frac{2}{\rho_{+}}\left(\frac{l^{2}+1}{l^{2}-1}\right)\right.} \\
& \left.+\frac{2}{\rho_{+}}\left(\frac{1+\gamma}{n^{2}}\right)\right] c_{1}+\left[-2 \frac{\rho_{+}+\gamma_{+}}{\rho_{+} n^{2}}-l \frac{l_{+}{ }^{2}+3}{l_{+}{ }^{2}-1}\right. \\
& \left.+\frac{4 l}{\rho_{+}\left(l^{2}-1\right)}+\frac{2}{\rho_{+}}\left(\frac{1+\gamma}{n^{2}}\right)\right] c_{3},
\end{aligned}
$$

where $\gamma=\gamma_{-}+\gamma_{+}$. If we let $\rho_{+}=1, \nu_{+}=\nu, \nu_{E-}=0$, and $\gamma_{-}=0$, we have the case of a single fluid, and from (19)(20) and (23) - (24) we can show that

$$
w=c_{1} e^{z}+c_{3} e^{l z}
$$

also applies for $0<z \leqslant d$. If $\nu_{E+}=0$, Eqs. (17)-(18) then become equivalent to

$$
\begin{gathered}
{\left[\begin{array}{cc}
2 & l^{2}+1 \\
n^{2}\left(l^{2}+1\right) /\left(l^{2}-1\right)+1+\gamma & 2 \ln ^{2} /\left(l^{2}-1\right)+1+\gamma
\end{array}\right]\left[\begin{array}{l}
c_{1} \\
c_{3}
\end{array}\right]} \\
=\left[\begin{array}{l}
0 \\
0
\end{array}\right]
\end{gathered}
$$

Equating the determinant of the $2 \times 2$ matrix of coefficients to zero, and defining $s=\nu^{1 / 2} l$ and $\epsilon=\nu^{1 / 2}$, so that $n=s^{2}-\epsilon^{2}$, we obtain the viscous surface gravity-capillary wave dispersion relation presented by Chandrasekhar, ${ }^{25}$

$$
\left(s^{2}+\epsilon^{2}\right)^{2}-4 s \epsilon^{3}+1+\gamma=0 .
$$

For $|\epsilon| \ll 1$ we obtain

$$
n= \pm i(1+\gamma)^{1 / 2}-2 \epsilon^{2}+O\left(\epsilon^{3}\right),
$$

so that the wave damping rate is indeed approximately $2 \nu k^{2}$ in terms of dimensional variables. ${ }^{10-12}$

In the general case, the coefficients of $c_{1}$ and $c_{3}$ which result when we substitute (19)-(20) and (23)-(24) into (17)-(18) are rather complicated expressions. If we assume that $d \ll 1$ and $\left|l_{+} d\right| \ll 1$, we obtain a simpler set of equations when we neglect terms containing $d^{2}$ and higher powers of $d$ in the Taylor expansion of the hyperbolic functions, and neglect terms of order $\nu_{E_{ \pm}} d$ in comparison with those of order $\nu_{E \pm}$ :

$$
\begin{gathered}
\left\{\frac{2}{\left(l^{2}-1\right)}+\frac{\nu_{E}}{n}+\left[-\frac{1-\rho_{+}+\gamma_{-}}{n^{2}}+\rho_{+} \frac{l_{+}{ }^{2}+3}{l_{+}{ }^{2}-1}-\frac{l^{2}+1}{l^{2}-1}\right.\right. \\
\left.\left.+\frac{\nu_{E+} \nu_{E-}\left(l_{+}{ }^{2}-1\right)}{\rho_{+} n^{2}}\right] d\right\} c_{1}+\left\{\frac{l^{2}+1}{l^{2}-1}+\frac{\nu_{E} l}{n}\right. \\
+\left[-\frac{1-\rho_{+}+\gamma_{-}}{n^{2}}+\rho_{+} l \frac{l_{+}{ }^{2}+3}{l_{+}{ }^{2}-1}-\frac{2 l}{\left(l^{2}-1\right)}\right. \\
\left.\left.+\frac{l \nu_{E+} \nu_{E-}\left(l_{+}{ }^{2}-1\right)}{\rho_{+} n^{2}}\right] d\right\} c_{3}=0,
\end{gathered}
$$

$$
\begin{gathered}
\left\{\frac{1+\gamma}{n^{2}}+\frac{l^{2}+1}{l^{2}-1}+\left[\frac{\rho_{+}+\gamma_{+}}{n^{2}}+\rho_{+}+\frac{2}{\left(l^{2}-1\right)}\right] d\right\} c_{1} \\
+\left\{\frac{1+\gamma}{n^{2}}+\frac{2 l}{\left(l^{2}-1\right)}+\left[l \frac{\rho_{+}+\gamma_{+}}{n^{2}}+\rho_{+}\right.\right. \\
\left.\left.+\frac{l^{2}+1}{l^{2}-1}\right] d\right\} c_{3}=0,
\end{gathered}
$$

where $\nu_{E}=\nu_{E+}+\nu_{E-}$. If $\nu_{E}=0$, and $d \rightarrow 0$, keeping $l_{+}$constant, we regain the relations (26)-(28) for a single viscous fluid.

The dispersion relation is again obtained by setting the determinant of the coefficient matrix to zero. Its behavior depends on the relative values of the parameters, particularly of $\nu_{+}$(hence $\left.l_{+}\right), d$, and $\nu_{E \pm}$.

\section{B. Very thin film}

If we let $d \rightarrow 0$, while letting $\nu_{+}$become large so that $\nu_{+} d \rightarrow$ const., we can neglect terms proportional to $d$ which do not have $l_{+}{ }^{2}-1$ in the denominator. We obtain

$$
\begin{aligned}
& {\left[2 \nu+\nu_{E}+4 \rho_{+} \nu_{+} d\right]\left[2 n \nu(1+n / \nu)^{1 / 2}+1+\gamma\right]} \\
& \quad-\left[n^{2}(1+2 \nu / n)+1+\gamma\right]\left[n+2 \nu+(1+n / \nu)^{1 / 2}\right. \\
& \left.\quad \times\left(\nu_{E}+4 \rho_{+} \nu_{+} d\right)\right]=0,
\end{aligned}
$$


which is of the same form as published dispersion relations for viscous surface films of negligible thickness, ${ }^{2-4,6-8}$ if we add $4 \rho_{+} \nu_{+} d$ to the non-dimensional viscosity of the film. More specifically, we should add it to the sum of the dilatational and shear viscosities of the film., ${ }^{5,27}$ Note that if $\nu_{+}$is allowed to be complex and frequency-dependent it will also make a contribution to the effective surface-film elasticity. A further discussion of the $\left(d \rightarrow 0, \nu_{+} d \rightarrow\right.$ const.) limit is given in Appendix B.

\section{Wave damping}

We can find a closed-form expression for the perturbation in the complex wave frequency due to the combined effect of the lower-fluid viscosity and the presence of the upper fluid layer, provided that the perturbation is relatively small. We assume that $n=-i \Gamma^{1 / 2}-\delta$, where $\Gamma=1+\gamma$, with $|\delta| \ll 1$ : the real part of $\delta$ is then the rate of wave damping. If we let $s=i^{-1 / 2} \Gamma^{1 / 4}(1+\alpha)$, defining $i^{\beta}=e^{i \pi \beta / 2}$, we have

$$
\delta=\epsilon^{2}+2 i \alpha \Gamma^{1 / 2},
$$

where we neglect higher-order terms in $\epsilon$ and $\alpha$. From (29) and (30), we obtain, neglecting higher-order corrections with respect to $d, \alpha$, and $\epsilon$ :

$$
\begin{aligned}
\left\{2 \epsilon^{2}+\nu_{T}+d\left[s^{2}\left(\rho_{+}-1\right)-\frac{1-\rho_{+}+\gamma_{-}}{s^{2}}\right]\right\} & \\
\times & {\left[\frac{1+\gamma}{s^{2}}+d \frac{\rho_{+}+\gamma_{+}}{\epsilon s}\right] } \\
= & {\left[1+\gamma+s^{4}+d\left(\rho_{+}+\gamma_{+}+\rho_{+} s^{4}\right)\right] } \\
& \times\left(1+\frac{\nu_{T}}{\epsilon s}+d \frac{\rho_{+} s}{\epsilon}\right)
\end{aligned}
$$

where

$$
\begin{aligned}
\nu_{T}= & \left(\chi_{+}+\chi_{-}\right) / n+\nu_{s+}+\nu_{s-}+4 \rho_{+} \nu_{+} d \\
& +\nu_{E+} \nu_{E-} d /\left(\rho_{+} \nu_{+}\right) .
\end{aligned}
$$

We thus have

$$
\begin{aligned}
\alpha \approx & \left\{-\frac{1}{2} i \Gamma^{-1 / 2} \epsilon^{2}-\frac{1}{4} i \Gamma^{-1 / 2} \nu_{T}+\frac{1}{2} \Gamma^{-1}\left[\gamma\left(1-\rho_{+}\right)\right.\right. \\
& \left.-\gamma_{-}\right] d-\frac{1}{4} i^{1 / 2} \rho_{+} d \epsilon^{-1} \Gamma^{-1 / 4} \nu_{T} \\
& \left.+\frac{1}{4} i^{-1 / 2}\left(\rho_{+} d\right)^{2} \epsilon^{-1} \Gamma^{1 / 4}\left(R^{2}-1\right)\right\} \\
& \times\left[1+i^{1 / 2} \epsilon^{-1} \Gamma^{-1 / 4} \nu_{T}+i^{-1 / 2} \rho_{+} d \epsilon^{-1} \Gamma^{1 / 4}\right]^{-1},
\end{aligned}
$$

where $R=\left(\rho_{+}+\gamma_{+}\right) /\left(\rho_{+} \Gamma\right)$, neglecting higher-order terms. Hence, to the same approximation,

$$
\begin{aligned}
\delta \approx & \left\{2 \epsilon^{2}+\frac{1}{2} \nu_{T}+i \Gamma^{-1 / 2}\left[\gamma\left(1-\rho_{+}\right)-\gamma_{-}\right] d\right. \\
& +\frac{1}{2} i^{-1 / 2} \rho_{+} d \epsilon^{-1} \Gamma^{1 / 4} \nu_{T}+\frac{1}{2} i^{1 / 2}\left(\rho_{+} d\right)^{2} \epsilon^{-1} \Gamma^{3 / 4} \\
& \left.\times\left(R^{2}-1\right)\right\}\left[1+i^{1 / 2} \epsilon^{-1} \Gamma^{-1 / 4} \nu_{T}\right. \\
& \left.+i^{-1 / 2} \rho_{+} d \epsilon^{-1} \Gamma^{1 / 4}\right]^{-1} .
\end{aligned}
$$

If $d \rightarrow 0$ this becomes identical to the corresponding formula for a film of negligible thickness. ${ }^{8,28}$ If $\nu_{E}=0$, i.e., we neglect surface and interfacial elasticity and viscosity, but if $\left|\nu_{+}\right| \gg \nu d^{-1}$, we have $\delta \approx 2^{-3 / 2}(1-i) \epsilon$, which reproduces the rate of damping for an inextensible film, ${ }^{4,29}$ and also Weber's ${ }^{23}$ result for a layer of grease ice which has such a high viscosity that the Stokes flow approximation can be applied in the upper layer.

The damping rate will be of order $\epsilon$ if either $d$ or $\nu_{T}$ are of the same order as or greater than $\epsilon$. If $\nu_{E}=0$ and $\left|\nu_{+} d\right| \geqslant O(\nu)$, but both $d$ and $\left|\nu_{+} d\right|$ are much smaller than $\nu^{1 / 2}$, we have $\delta \approx 2 \epsilon^{2}+2 \rho_{+} \nu_{+} d$. If the densities of the upper and lower fluids are equal $\left(\rho_{+}=1\right)$ and $\gamma_{+}=\gamma_{-}=0$, this is consistent with the damping rate for a fluid with nondimensional viscosity which varies more-or-less arbitrarily with depth ${ }^{30,31}$ but which is always much smaller than 1 :

$$
\delta \approx 2 \int_{-\infty}^{0} \nu(z) 2 e^{2 z} d z
$$

Keeping $\nu_{E}=0$, if $d$ is so large that $\frac{1}{2} i^{-1 / 2} \rho_{+} d \epsilon^{-1} \Gamma^{1 / 4}\left(4 \rho_{+} \nu_{+} d\right)$ dominates over the other contributions to the numerator of (36), and the denominator is dominated by its final term, we find that $\operatorname{Re} \delta \approx 2 \rho_{+} \nu_{+} d$ if $\rho_{+}=1$ and $\gamma_{-}=0$. This is also consistent with (37).

\section{Numerical example}

In order to get some idea of the relative sizes of the various contributions to (36), we look firstly at waves of 2 $\mathrm{cm}$ wavelength on a system containing a lower fluid with density $1000 \mathrm{~kg} \mathrm{~m}^{-3}$ and kinematic viscosity $10^{-6} \mathrm{~m}^{2} \mathrm{~s}^{-1}$ (corresponding to water), and an upper fluid with density $900 \mathrm{~kg} \mathrm{~m}^{-3}$ and viscosity $10^{-4} \mathrm{~m}^{2} \mathrm{~s}^{-1}$ (corresponding to a heavy fuel oil). We assume that the surface tension is $25 \mathrm{mN} \mathrm{m}^{-1}$, the interfacial tension is $15 \mathrm{mN} \mathrm{m}^{-1}$, and the thickness of the upper layer is $0.1 \mathrm{~mm}$. We assume that the surface elasticity is $15 \mathrm{mN} \mathrm{m}^{-1}$, the interfacial elasticity is $10 \mathrm{mN} \mathrm{m}^{-1}$, and that the surface and interfacial viscosities can be neglected. (The assumed surface and interfacial elasticities are at the lower end of the typical range ${ }^{32} 10-50$ $\mathrm{mN} \mathrm{m}^{-1}$ for surfactant materials on water.) The $a k \ll 1$ requirement means that the wave amplitude should be much smaller than $3 \mathrm{~mm}$, but it is permitted to be greater than the upper-layer thickness.

The various nondimensional parameters are then as follows: 


$$
\begin{aligned}
& \rho_{+}=0.9, \quad \gamma_{+}=0.2516, \quad \gamma_{-}=0.1510, \\
& 1+\gamma=1.403, \quad \chi_{+}=0.1510, \quad \chi_{-}=0.1006, \\
& \nu=0.001778, \quad \nu_{+}=0.1778, \quad \epsilon=0.04216, \\
& l_{+} \approx 2.17-1.93 i, \quad d=0.03142, \\
& l_{+} d \approx 0.0682-0.0605 i .
\end{aligned}
$$

The contributions to $\nu_{T}$ are $\chi_{+} / n \approx 0.1275 i$, $\chi_{-} / n \approx 0.0850 i, 4 \rho_{+} \nu_{+} d=0.0201$, and $\nu_{E+} \nu_{E-} d /\left(\rho_{+} \nu_{+}\right)$ $\approx-0.0021$. We see that in this case, $4 \rho_{+} \nu_{+} d$ is an order of magnitude smaller than $\chi_{+} / n+\chi_{-} / n$, and that the final term $\nu_{E+} \nu_{E-} d /\left(\rho_{+} \nu_{+}\right)$is an order of magnitude even smaller. An increase of a factor 10 in $\nu_{+}$will bring $4 \rho_{+} \nu_{+} d$ up to the same order of magnitude as the surface elasticity contribution. The final term may possibly become significant for $\nu_{+}$in the range $d^{2} \ll \nu_{+} \ll 1$.

The various contributions to (36) are

$$
\begin{aligned}
& \rho_{+} d \epsilon^{-1}=0.6705, \quad\left(\rho_{+} d\right)^{2} \epsilon^{-1}=0.01896, \\
& 2 \epsilon^{2}=0.003556, \quad \frac{1}{2} \nu_{\mathrm{T}}=0.00909+0.1062 i, \\
& i \Gamma^{-1 / 2}\left[\gamma\left(1-\rho_{+}\right)-\gamma_{-}\right] d=-0.00294 i, \\
& \frac{1}{2} i^{-1 / 2} \rho_{+} d \epsilon^{-1} \Gamma^{1 / 4} \nu_{\mathrm{T}} \approx 0.0594+0.0502 i, \\
& \frac{1}{2} i^{1 / 2}\left(\rho_{+} d\right)^{2} \epsilon^{-1} \Gamma^{3 / 4}\left(R^{2}-1\right)=-0.00145-0.00145 i, \\
& i^{1 / 2} \Gamma^{-1 / 4} \nu_{\mathrm{T}} \epsilon^{-1} \approx-3.00+3.55 i,
\end{aligned}
$$

and

$$
i^{-1 / 2} \rho_{+} d \epsilon^{-1} \Gamma^{1 / 4}=0.516-0.516 i .
$$

We find that $\delta \approx 0.0313-0.0385 i$, which is of the same order of magnitude as $\epsilon$, and is dominated by the effect of the surface and interfacial elasticities.

Figures 1(a)-1(h) show the damping ratio $y(f)$, the computed wave damping rate divided by the damping rate for pure water, as a function of frequency $f=\omega /(2 \pi)$. Using non-dimensional variables, $y(f)=\frac{1}{2} \epsilon^{-2} \operatorname{Re} \delta$. This ratio is plotted for eight different cases, covering three different viscosity ratios $\nu_{+} / \nu_{-}$, and the presence and absence of surface/interfacial elasticities $\chi_{ \pm}$and of interfacial tension $\gamma_{-}$. For each case, results for various thicknesses of the upper fluid layer are plotted. The other parameters are as specified previously. The frequency corresponding to the wavelength of $2 \mathrm{~cm}$ is also indicated. The damping ratio for a surfactant film of oleic acid on water of surface tension $73 \mathrm{mN} \mathrm{m}^{-1}$, from Ref. 8, is also shown in all plots for reference.

It can be seen that the effect of surface/interface elasticity generally dominates the wave damping, the exceptions being for $\nu_{+} / \nu_{-}=1000$ with (dimensional) $d>1 \mathrm{~mm}$, and for $\nu_{+} / \nu_{-}=10000$ with $d>30 \mu \mathrm{m}$. Nevertheless, the damping is affected significantly by the finite thickness of the film when $\nu_{+} / \nu_{-}=100$ and $d \geqslant 1 \mathrm{~mm}$. If the upper layer is extremely viscous, we approach the rigid-film limit with $y(f)=2^{-5 / 2} \epsilon^{-1}$, which is proportional to $f^{-3 / 2}$ in the low- frequency limit. For a fixed value of $\nu_{+}$, at a sufficiently low frequency the rigid-film approximation will break down, which explains why even at $\nu_{+} / \nu_{-}=10000$ we see a peak in the $y(f)$ curves.

The effect of removing the interfacial tension and elasticity, as may be expected if an oil/water interface becomes emulsified, does not produce any qualitative difference in the wave damping [see Figs. 1(c) and 1(d)]. The main effects are probably due to changes in the dispersion relation as a result of the reduced value of $\gamma=\gamma_{+}+\gamma_{-}$in the case of zero surface/interfacial elasticity, and due to the reduction in total surface/interfacial elasticity $\chi=\chi_{+}+\chi_{-}$where the surface elasticity is non-zero.

\section{CONCLUSION}

In this paper we have derived a simple approximate expression for the damping rate (and associated frequency shift) of linear surface gravity-capillary waves due to the presence of a thin layer of a Newtonian fluid on the surface of a Newtonian fluid of infinite depth. The effects of surface and interfacial elasticity and viscosity are taken into account. The vertical variation of the fluid motions within each fluid layer is described exactly by exponential functions: it is not necessary to use approximations to the governing ordinary differential equation (14). Applying the surface and interfacial boundary conditions leads to two linear simultaneous equations in two unknowns which have coefficients containing hyperbolic functions of the upper layer thickness. Equating the determinant of the coefficients to zero leads to a rather complicated transcendental equation for the wave dispersion relation; considerable simplification is achieved if the upper fluid layer is sufficiently thin $(k d \ll 1$ and $\left|\omega \nu_{+}{ }^{-1}\right|^{1 / 2} d \ll 1$ in dimensional units).

The dispersion relation for a "monomolecular' viscoelastic film of essentially zero thickness ${ }^{4,6-8}$ is reproduced if we let $d \rightarrow 0$, if we replace the surface tension, elasticity and viscosity by the sums of the surface and interfacial values of the respective quantities, and then add $4 \rho_{+} \nu_{+} d$ to the surface viscosity. Additional effects of finite surface-layer thickness also arise due to the fluid density difference and interfacial tension. Calculations of wave damping rates under conditions representative of slicks of heavy fuel oil on water indicate that the effect of surface elasticity generally dominates the wave damping, but that the finite surface-layer thickness causes significant changes in the damping if the surface layer is $100 \mu \mathrm{m}$ thick or greater.

It should be possible to use the theory presented in this paper to compute from first principles the effect of oil slicks of finite thickness in damping wind-generated waves, given accurate laboratory data on viscosity, surface tension and interfacial tension. It is still necessary to take account of the effect of surfactants which are present both in the oil and in the surrounding water, ${ }^{13,33}$ as the effect of surface/interfacial elasticity dominates the wave damping under normal circumstances.

The wave damping rate approximation is also consistent with previously published results for damping due to vertically-varying (eddy) viscosity, ${ }^{30,31}$ and due to a surface 
viscous or viscoelastic fluid layer representing grease ice. ${ }^{23}$

For applications such as distinguishing oil slicks from natural films by their radar backscatter characteristics, ${ }^{17-22}$ it will also be necessary to consider in detail the atmospheric boundary layer processes leading to wave generation by wind forcing, and the nonlinear interaction processes between the different Fourier components of the surface-wave field. ${ }^{7,8,34-37}$
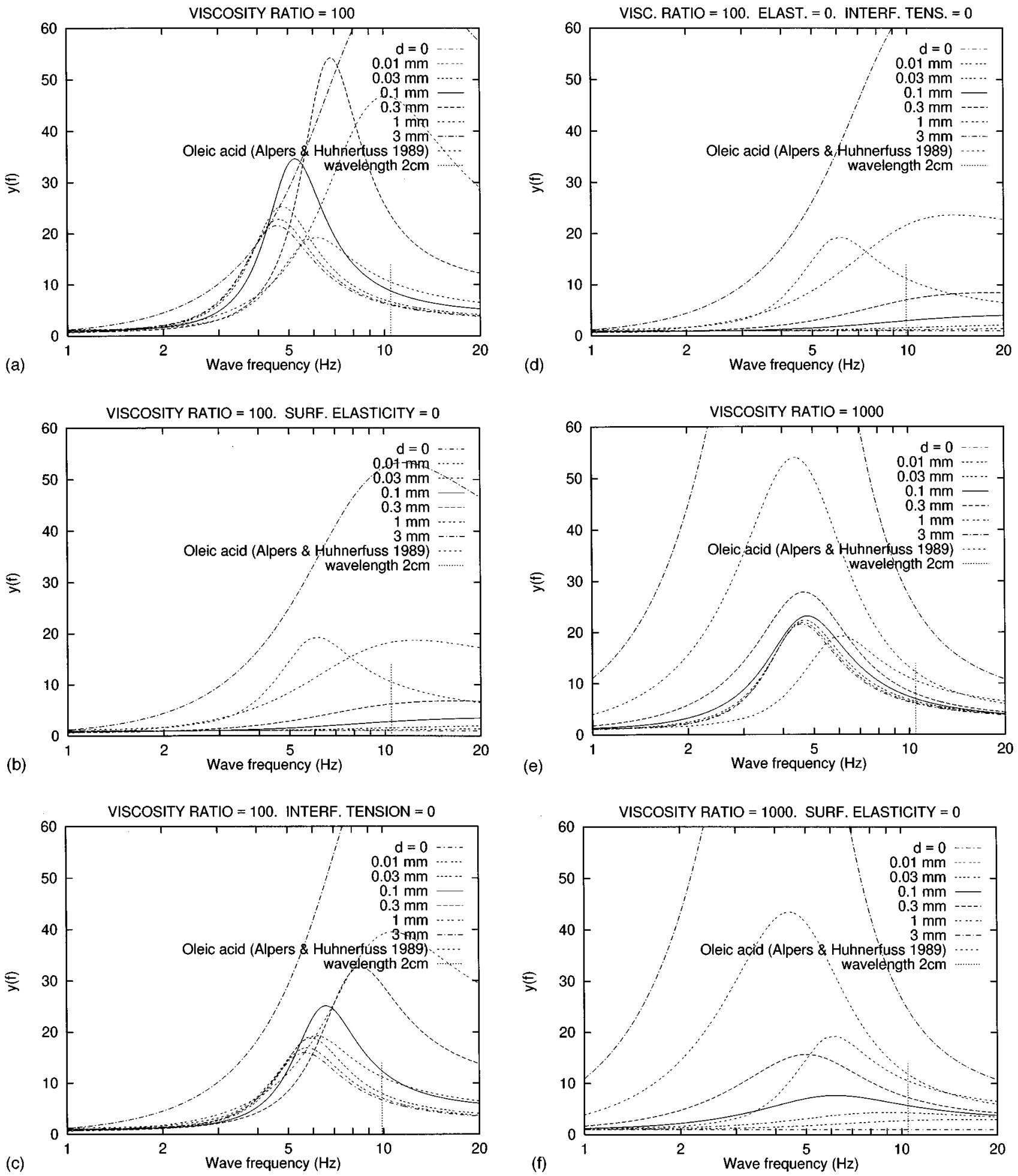

FIG. 1. The ratio, $y(f)$, of the wave damping rate to the damping rate for a single fluid (water) with an uncontaminated surface, as a function of frequency $f$, for various thicknesses of the upper fluid. The thicknesses corresponding to the different line types are shown in the key. The sub-figure headings show the viscosity ratio $\nu_{+} / \nu_{-}$, and whether either or both of: (i) the surface/interfacial elasticity $\chi_{ \pm}$; (ii) the interfacial tension $\gamma_{-}$and also the elasticity $\chi_{-}$; are set to zero. Other physical parameters are as specified in Sec. III D. The corresponding values of $y(f)$ for an oleic acid surfactant film on water, from Alpers and Hühnerfuss (see Ref. 8) are shown for reference on each sub-figure, and also a vertical line at the frequency corresponding to a wavelength of $2 \mathrm{~cm}$. 

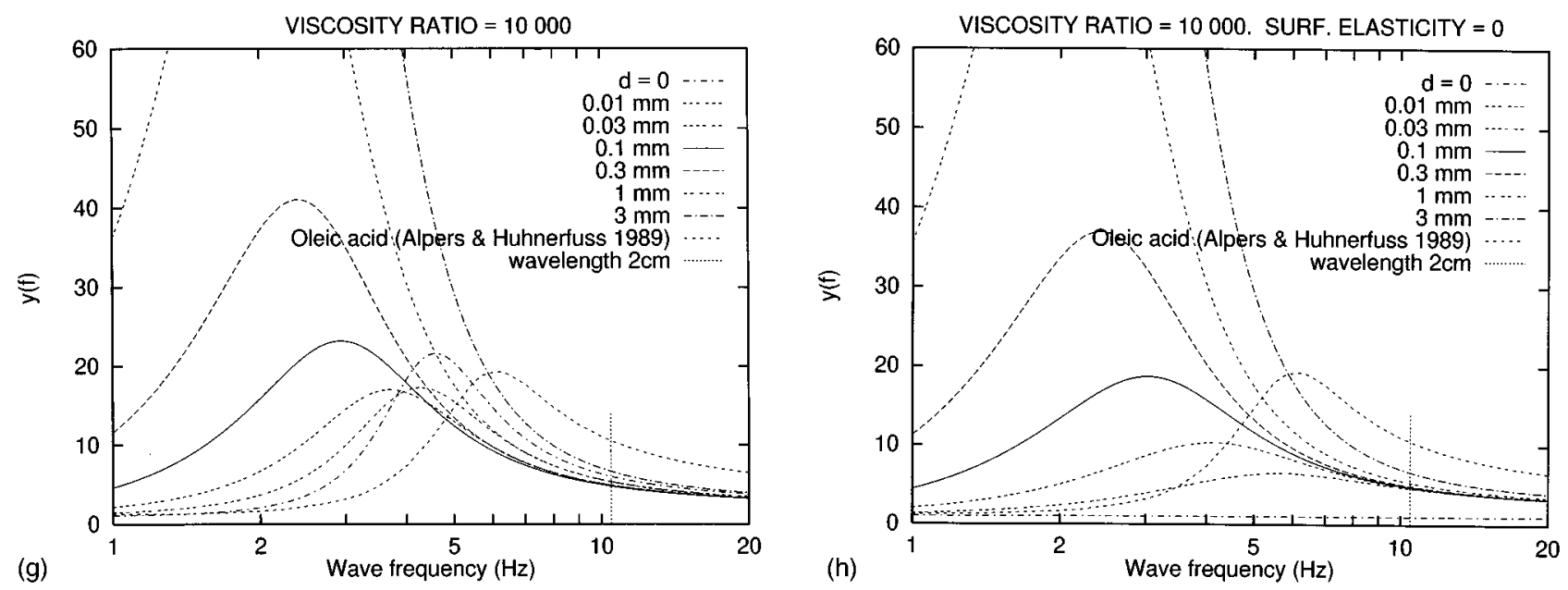

FIG. 1. (Continued.)

\section{ACKNOWLEDGMENTS}

The authors thank Professor Kristian B. Dysthe and Heidi A. Espedal for constructive criticism and useful discussions. Constructive criticism from two anonymous referees was very helpful in improving the manuscript. A.D.J. was supported by the Research Council of Norway under the project "Synthetic aperture radar analysis and modelling for ocean monitoring.' S.J.J. was supported by the U.S. Office of Naval Research under the project "'Ocean surface processes and remote sensing," contract N00014-92-J1650.

\section{APPENDIX A: NONLINEAR EFFECTS}

In this section we treat nonlinear effects, with viscosity and surface tension neglected. Again taking $\varpi$ as the actual pressure, we let

$$
\begin{aligned}
& \varpi_{+}=\rho_{+}\left(P_{+}-g z\right)+g \rho_{+} d, \\
& \varpi_{-}=\rho_{-}\left(P_{-}-g z\right)+g \rho_{+} d,
\end{aligned}
$$

where the subscripts refer to the layers, and we scale the variables through

$$
\begin{aligned}
& \left(x^{*}, z^{*}\right)=(x, z) k, \quad t^{*}=(g k)^{1 / 2} t, \\
& \left(U^{*}, W^{*}\right)=(1 / \Delta)(g / k)^{-1 / 2}(U, W), \quad P^{*}=[k /(g \Delta)] P,
\end{aligned}
$$

$$
Z^{*}=(k / \Delta) Z, \quad H^{*}=(k / \Delta) H, \quad d^{*}=k d, \quad \rho^{*}=\rho_{+} / \rho_{-},
$$

where $\Delta$ is a small parameter. Then, omitting the asterisks and letting $\theta=x-c t$, the equations governing irrotational flow take the form

$$
\begin{aligned}
& \frac{\partial U}{\partial \theta}+\frac{\partial W}{\partial z}=0, \\
& \frac{\partial}{\partial \theta}\left[P+(\Delta / 2)\left(U^{2}+W^{2}\right)-c U\right]=0, \\
& -c \frac{\partial W}{\partial \theta}+\frac{\partial}{\partial z}\left[P+(\Delta / 2)\left(U^{2}+W^{2}\right)\right]=0,
\end{aligned}
$$

where we assume that the motion is steady when expressed in terms of the coordinates $(\theta, z)$. The boundary conditions are

$$
W_{+}=-c \frac{\partial Z}{\partial \theta}+\Delta U_{+} \frac{\partial Z}{\partial \theta}, \quad P_{+}=Z,
$$

at $z=d+\Delta Z$, and

$$
W_{ \pm}=-c \frac{\partial H}{\partial \theta}+\Delta U_{ \pm} \frac{\partial Z}{\partial \theta}, \quad P_{-}-\rho P_{+}=(1-\rho) H,
$$

at $z=\Delta H$.

We now let $F$ denote $U, W, P, Z$, or $H$, and we expand $F$ and $c$ in the form

$$
\begin{aligned}
& F=F^{(0)}+\Delta F^{(1)}+\Delta^{2} F^{(2)}+\ldots, \\
& c=c^{(0)}+\Delta^{2} c^{(2)}+\ldots,
\end{aligned}
$$

as in the Stokes expansion for waves on the surface of a homogeneous fluid. ${ }^{38}$ Our aim here is to determine the effects of nonlinearity when the upper layer depth $d$ is small.

Taking $H^{(0)}=A \cos \theta$, we find that

$$
\begin{aligned}
& U_{ \pm}^{(0)}=A\left(\alpha_{ \pm} \cosh z+\beta_{ \pm} \sinh z\right) \cos \theta, \\
& P_{ \pm}^{(0)}=c^{(0)} U_{ \pm}^{(0)}, \\
& W_{ \pm}^{(0)}=A\left(\alpha_{ \pm} \sinh z+\beta_{ \pm} \cosh z\right) \sin \theta,
\end{aligned}
$$

where

$$
\alpha_{-}=\beta_{-}=1, \quad \alpha_{+}=\frac{\left(c^{(0)}\right)^{2}-(1-\rho)}{\rho c^{(0)}}, \quad \beta_{+}=c^{(0)} .
$$

Here $c^{(0)}$ solves

$$
\left[\left(c^{(0)}\right)^{2}-1\right]\left[\left(c^{(0)}\right)^{2}-\frac{(1-\rho) \sinh d}{\cosh d+\rho \sinh d}\right]=0,
$$

a dimensionless form of a well-known equation. ${ }^{39}$ There are two solutions describing waves travelling in the positive $x$ direction, the barotropic mode, $c^{(0)}=1$, and the baroclinic 
mode, for which the second factor in (A11) vanishes. For the latter mode the wave speed is $O\left(d^{1 / 2}\right)$ in the case $d \ll 1$.

Our calculation of wave decay is concerned exclusively with the barotropic mode, and we therefore restrict our attention to this mode by taking $c^{(0)}=1$. Then the $O(1)$ solution becomes

$$
\begin{aligned}
& U_{ \pm}^{(0)}=P_{ \pm}^{(0)}=A e^{z} \cos \theta, \quad W_{ \pm}^{(0)}=A e^{z} \sin \theta, \\
& Z^{(0)}=A e^{d} \cos \theta, \quad H^{(0)}=A \cos \theta,
\end{aligned}
$$

and a short calculation shows that the $O(\Delta)$ solution is

$$
\begin{aligned}
& U_{ \pm}^{(1)}=W_{ \pm}^{(1)}=0, \quad P_{ \pm}^{(1)}=-\frac{1}{2} A^{2} e^{2 z} \\
& Z^{(1)}=\frac{1}{2} A^{2} e^{2 d} \cos 2 \theta, \quad H^{(1)}=\frac{1}{2} A^{2} \cos 2 \theta .
\end{aligned}
$$

Using (A12) and (A13), we find that the $O\left(\Delta^{2}\right)$ equations can be reduced to

$$
\frac{\partial^{2} W_{ \pm}^{(2)}}{\partial \theta^{2}}+\frac{\partial^{2} W_{ \pm}^{(2)}}{\partial z^{2}}=0
$$

with boundary conditions

$$
W_{+}^{(2)}-\frac{\partial W_{+}^{(2)}}{\partial z}=\left(2 c^{(2)}-A^{2} e^{2 d}\right) A e^{d} \cos \theta,
$$

at $z=d$, and

$$
W_{ \pm}^{(2)}-\frac{1}{1-\rho}\left[\frac{\partial W_{-}^{(2)}}{\partial z}-\rho \frac{\partial W_{+}^{(2)}}{\partial z}\right]=\left(2 c^{(2)}-A^{2}\right) A \cos \theta
$$

at $z=0$. The solution takes the form

$$
\begin{aligned}
& W_{-}^{(2)}=A \alpha e^{z} \sin \theta \\
& W_{+}^{(2)}=A(\beta \cosh z+\gamma \sinh z) \sin \theta
\end{aligned}
$$

where $\alpha, \beta$, and $\gamma$ are constants.

Substituting (A17) into the boundary conditions yields three linear equations for these constants, for which the determinant of the coefficients vanishes. A solvability condition is required, and is given by

$$
c^{(2)}=\frac{1}{2}\left[\frac{1+\rho\left(e^{4 d}-1\right)}{1+\rho\left(e^{2 d}-1\right)}\right] A^{2},
$$

which reduces to the standard solution ${ }^{38}$ in the case $d \rightarrow 0$. As can be seen, the perturbation expansion (A8) remains wellordered in this limit, and therefore it appears that the linearization procedured employed earlier is valid for $d \ll 1$. In particular, it is valid for $d \ll \Delta$, so that the wave amplitude is permitted to be much greater than the upper-layer thickness.

The linearization procedure should also be valid in the case where we include fluid viscosity. This is certainly the case for waves on a single fluid which have $a k \ll 1$ but where $a$ can exceed the thickness $(2 \nu / \omega)^{1 / 2}$ of the viscous surface boundary layer. One way to show this is to employ a Lagrangian description of the hydrodynamic equations, for which the $O(a k)$ perturbation allows $a$ to exceed the boundary-layer thickness without any conceptual difficulties, although it is formally identical to the $O(a k)$ perturbation in the Eulerian description. ${ }^{40,41}$

Finally, the fact that we have imposed boundary conditions at the mean surface can be justified with reference to Miles. ${ }^{42}$ In these studies of the generation of surface waves by shear flows, he found that imposing the boundary condition at the mean surface rather than at the surface wave made no difference to the results.

\section{APPENDIX B: LIMIT AS $\boldsymbol{d} \rightarrow 0$}

If interfacial surfactant effects are omitted and if we consider a thin upper layer, as in Sec. III B, the dimensional dynamic boundary conditions at $z=0$ become

$$
\begin{aligned}
& p-2 \nu_{-} \frac{\partial w}{\partial z}=\left[g+\frac{k^{2}}{\rho_{-}}\left(\gamma_{+}+\gamma_{-}\right)\right] H, \\
& \left(4 \rho_{+} \nu_{+} k^{2} d\right) u+\rho_{-} \nu_{-}\left(\frac{\partial u}{\partial z}+i k w\right)=0,
\end{aligned}
$$

with errors of $O(d)$. Equations (B1) and (B2) were obtained by taking the small- $d$ limit of the exact solution, with $\nu_{+} d$ fixed, and were checked by letting $\zeta=z / d$ denote a scaled variable and by solving for the flow in the upper layer through the use of an ordinary perturbation series in $d$.

For the flow of a single layer of fluid covered by an insoluble surface film at $z=H$, Miles, ${ }^{26}$ generalizing earlier work by Dorrestein, ${ }^{2}$ assumes a tangential surface force

$$
\nabla_{s} \gamma+\eta_{1} \nabla_{s}\left(\nabla_{s} \cdot \mathbf{u}_{s}\right)+\eta_{2} \nabla_{s}^{2} \mathbf{u}_{s},
$$

where $\gamma$ is the surface tension, $\nabla_{s}$ is the surface gradient, $\mathbf{u}_{s}$ is the tangential velocity at the surface, and $\eta_{1}$ and $\eta_{2}$ are surface viscosity coefficients. Miles also defines $\Gamma$ as the concentration of the surface film, with reference value $\Gamma_{0}$, and $\chi$ as the surface elasticity, with mathematical definition

$$
\chi=-\Gamma_{0}(d \gamma / d \Gamma)_{\Gamma=\Gamma_{0}} .
$$

When expressed in terms of the present notation, Miles' theory shows that the linearized normal and tangential boundary conditions for two-dimensional flow in the $x z$-plane are

$$
p-2 \nu_{-} \frac{\partial w}{\partial z}=\left(g+\frac{k^{2}}{\rho_{-}} \gamma\right) H,
$$

and

$$
\left(\frac{\chi}{n}+\eta_{1}+\eta_{2}\right) k^{2} u+\rho_{-} \nu_{-}\left(\frac{\partial u}{\partial z}+i k w\right)=0,
$$

where the sum $\eta_{1}+\eta_{2}$ plays the role of the surface viscosities $\nu_{s \pm}$ in (5) and (10). Hence, by comparing (B1) and (B5), and $(\mathrm{B} 2)$ and $(\mathrm{B} 6)$, we see that a thin $(d \rightarrow 0)$ but very viscous $\left[\nu_{+}=O\left(d^{-1}\right)\right]$ upper layer of Newtonian fluid has exactly the same properties as regards wave decay as an insoluble surface film if we replace $\gamma$ in the surface film theory by $\gamma_{+}+\gamma_{-}$, and $\left(\chi / n+\eta_{1}+\eta_{2}\right)$ in the surface film theory by $4 \rho_{+} \nu_{+} d$. 
${ }^{1}$ C. Marangoni, "Sul principio della viscosità superficiale dei liquidi stabili,'” Nuovo Cimento, Ser. 2 5/6, 239 (1872).

${ }^{2}$ R. Dorrestein, "General linearized theory of the effect of surface films on water ripples," Proc. K. Ned. Akad. Wet., Ser. B 54, 260 (1951).

${ }^{3}$ V. G. Levich, Physicochemical Hydrodynamics (Prentice-Hall, Englewood Cliffs, NJ, 1962), Chap. 11.

${ }^{4} \mathrm{M}$. van den Tempel and R. P. van de Riet, "Damping of waves by surfaceactive materials," J. Chem. Phys. 42, 2769 (1965).

${ }^{5}$ J. W. Miles, "Surface-wave damping in closed basins," Proc. R. Soc. London, Ser. A 297, 459 (1967).

${ }^{6} \mathrm{E}$. H. Lucassen-Reynders and J. Lucassen, "Properties of capillary waves," Adv. Colloid Interface Sci. 2, 347 (1969).

${ }^{7} \mathrm{~K}$. Dysthe and Y. Rabin, "Damping of short waves by insoluble surface films," in Proceedings of ONRL Workshop, Report C-11-86 (U.S. Office of Naval Research, London, 1986), pp. 187-213.

${ }^{8} \mathrm{~W}$. Alpers and H. Hühnerfuss, "The damping of ocean waves by surface films: A new look at an old problem,"' J. Geophys. Res. 94, 6251 (1989).

${ }^{9}$ G. G. Stokes, Mathematical and Physical Papers (Cambridge University Press, Cambridge, England, 1880).

${ }^{10}$ G. G. Stokes, "On the theories of the internal friction of fluids in motion, and of the equilibrium and motion of elastic solids, Trans. Cambridge Philos. Soc. 8, 287 (1845) (See Ref. 9, Vol. 1 pp. 75-129).

${ }^{11}$ G. G. Stokes, "On the effect of the internal friction of fluids on the motion of pendulums," Trans. Cambridge Philos. Soc. 9, 8 (1850) (see Ref. 9, Vol. 3, pp. 1-141)

${ }^{12}$ H. Lamb, Hydrodynamics, 6th ed. (Cambridge University Press, Cambridge, England, 1932), pp. 625-628.

${ }^{13}$ D. P. Hoult, "'Oil spreading on the sea," Annu. Rev. Fluid Mech. 44, 341 (1972).

${ }^{14}$ J. Buckmaster, "Viscous-gravity spreading of an oil slick," J. Fluid Mech. 59, 481 (1973).

${ }^{15}$ K. S. Aravamudan, P. K. Raj, and G. Marsh, "Simplified models to predict the breakup of oil on rough seas," in Proceedings of the 1981 Oil Spill Conference, March 2-5, 1981, Atlanta, Georgia (American Petroleum Institute, Washington, DC, 1981), pp. 153-159; M. S. Belen, W. J. Lehr, and H. M. Cekirge, "Spreading, dispersion, and evaporation of oil slicks in the Arabian Gulf, ibid., pp. 161-164.

${ }^{16}$ L. N. Persen, "On the physical behaviour of an oil spill at sea," Technical report, Institute of Mechanics, The Technical University of Norway, Trondheim, 1981 (Available from Norges Tekniske Universitetsbibliotek, Trondheim, Norway, URL http://www.bibsys.no/bibsys.html).

${ }^{17} \mathrm{H}$. Hühnerfuss, W. Alpers, and K. Richter, "Discrimination between crude-oil spills and monomolecular sea slicks by airborne radar and infrared radiometer-possibilities and limitations," Int. J. Remote Sensing 7, 1001 (1986)

${ }^{18}$ T. Iguchi and H. Inomata, "SIR-B experiment in Japan: IV Experimental results; 3. Oil pollution experiment," J. Radio Res. Lab. 35 (Special Issue No. 2), 85 (1988).

${ }^{19}$ H. A. Hovland-Espedal, J. A. Johannessen, and G. Digranes, "Norwegian surface slick report," Technical Report No. 81, Nansen Environmental and Remote Sensing Center, Bergen, Norway, 1994 (Available from Nansen Environmental and Remote Sensing Center, email administrasjon@nrsc.no); “Slick detection in SAR images," in Proceedings, IGARSS'94, Pasadena, California (IEEE, Pisacataway, NJ, 1994), Vol. 4, pp. 2038-2040.

${ }^{20} \mathrm{H}$. Hühnerfuss et al., "Classification of sea slicks by multifrequency radar techniques: New chemical insights and their geophysical implications," J. Geophys. Res. 99, 9835 (1994).

${ }^{21}$ V. Wismann, M. Gade, W. Alpers, and H. Hühnerfuss, "Radar signatures of marine mineral oil spills measured by an airborne multifrequency radar," J. Geophys. Res. (submitted).

${ }^{22}$ H. A. Espedal, O. M. Johannessen, and J. C. Knulst, "Natural films in coastal waters," in Proceedings, IGARSS'95, Florence, Italy, July 10-14, 1995 (IEEE, Pisacataway, NJ, 1995), pp. 2106-2108.

${ }^{23}$ J. E. Weber, "Wave attenuation and wave drift in the marginal ice zone," J. Phys. Oceanogr. 17, 2351 (1987).

${ }^{24}$ D. D. Joseph, Fluid Dynamics of Viscoelastic Liquids (Springer-Verlag, New York, 1990).

${ }^{25} \mathrm{~S}$. Chandrasekhar, Hydrodynamic and Hydromagnetic Stability (Oxford University Press, Oxford, England, 1961), p. 451.

${ }^{26} \mathrm{~J}$. W. Miles, "A note on surface films and surface waves," Wave Motion 13, 303 (1991).

${ }^{27}$ A. D. Jenkins and K. B. Dysthe, "The effective film viscosity coefficients of a thin floating fluid layer," J. Fluid Mech. (submitted).

${ }^{28}$ See Ref. 7, Eq. 17.

${ }^{29}$ O. Reynolds, "On the effect of oil in destroying waves on the surface of water," Brit. Assoc. Adv. Sci. Rep. (1880) (Papers, Vol. 1, p. 409, see Ref. 12, p. 631).

${ }^{30}$ S. V. Dobroklonskii and N. V. Kontoboitseva, "An experimental investigation of turbulent viscosity in monochromatic waves," Izv. Akad. Nauk SSSR, Fiz. Atmos. Okeana 2, 64 (1966) [Izv. Acad. Sci. USSR, Atmos. Oceanic Phys. 2, 35 (1966)].

${ }^{31} \mathrm{~A}$. D. Jenkins, "Wind and wave induced currents in a rotating sea with depth-varying eddy viscosity," J. Phys. Oceanogr. 17, 938 (1987).

${ }^{32}$ S. Hård and R. D. Neuman, "Laser light-scattering measurements of viscoelastic monomolecular films," J. Colloid Interface Sci. 83, 315 (1981); F. M. Snik, W. J. J. Kouijzer, J. C. M. Keltjens, and Z. Houkes, "The measurement of dilatational properties of liquid surfaces," ibid. 93, 310 (1983).

${ }^{33}$ J. A. Fay, "Physical processes in the spread of oil on a water surface," in Conference on Prevention and Control of Oil Spills, Sponsored by API, EPA, and U.S. Coast Guard, Washington, DC, 1971 (see Ref. 13).

${ }^{34}$ L. M. Fitzgerald, "Wind-induced stresses on water surfaces," Aust. J. Phys. 16, 475 (1963).

${ }^{35}$ J. C. Gottifredi and G. J. Jameson, "The suppression of wind-generated waves by a surface film," J. Fluid Mech. 32, 609 (1968).

${ }^{36}$ T. R. Larson and J. W. Wright, "Wind-generated gravity-capillary waves: Laboratory measurements of temporal growth rates using microwave backscatter," J. Fluid Mech. 70, 417 (1975).

${ }^{37} \mathrm{X}$. Zhang, "Capillary-gravity and capillary waves generated in a wind wave tank: Observations and theories,', J. Fluid Mech. 289, 51 (1995).

${ }^{38}$ See Ref. 12, p. 417.

${ }^{39}$ P. K. Kundu, Fluid Mechanics (Academic, New York, 1990), pp. 229233.

${ }^{40}$ Ümit Ünlüata and C. C. Mei, "Mass transport in water waves,' J. Geophys. Res. 75, 7611 (1970).

${ }^{41}$ J. E. Weber, "Steady wind- and wave-induced currents in the open ocean," J. Phys. Oceanogr. 15, 936 (1983).

${ }^{42} \mathrm{~J}$. W. Miles, "On the generation of surface waves by shear flows," J. Fluid Mech. 3, 185 (1957); "On the generation of surface waves by shear flows. Part 2," ibid. 6, 568 (1959). 